\title{
ManejoSoja3D: Ambiente Virtual para Aprendizado de Manejo da Cultura da Soja
}

\author{
Daniel Souza Teixeira1, Vanessa Xavier Camargo', Emília Alves Nogueira1, \\ Marcos Wagner S. Ribeiro 1 \\ ${ }^{1}$ Instituto de Ciências Exatas Universidade Federal de Goiás (UFG) - Regional Jataí \\ Jataí GO Brasil \\ daniel.fnz@hotmail.com, \{emiliacd8, vanessaxcamargo\}@gmail.com, \\ marcos_ribeiro@ufg.br
}

\begin{abstract}
This paper presents a methodology based on the L-System plant growth method to simulate the behavior of the soybean crop in the alteration of soil macronutrients, having a virtual environment to support the visualization of these interactions.
\end{abstract}

Resumo. Este artigo apresenta uma metodologia baseada no método de crescimento de plantas L-System para simular o comportamento da cultura da soja em meio a alteração de macronutrientes do solo tendo um ambiente virtual como suporte a visualização destas interações.

\section{Introdução}

O curso de agronomia e áreas afins possuem disciplinas relacionadas com o manejo de algumas das principais culturas existentes no país. A principal dificuldade encontrada por docentes e consequentemente pelos discentes destes conteúdos é a exemplificação e o acompanhamento in loco do cultivar. Estes problemas ocorrem por várias razões, como: a) não enquadramento do período de plantação e da oferta da disciplina; b) tempo necessário para que cada fase da cultura tenha maturidade e ofereça resultados; c) diversidade de variáveis que influenciam no manejo da cultura e que necessitam ser estudadas isoladamente. Além destes aspectos, a dificuldade de se obter um processo eficiente de plantio e manejo de uma cultura está diretamente ligado ao não entendimento do papel de uma análise de solo, antes de realizar o plantio. Outros fatores como desconhecimento das principais as fases de crescimento da planta, influências de macro e micronutrientes, também podem atrapalhar o aprendizado, seja para compor o solo, combater pragas ou realizar uma análise de desempenho. Diante isso, a utilização de recursos computacionais como a simulação por meio do uso de Ambientes Virtuais (AV) podem auxiliar, fornecendo, meios de estudos, experimentação, análises e avaliações de situações que seriam inviáveis de se realizar no mundo real, pois, meios tradicionais demandariam muito tempo e muitos recursos financeiros. Além de uma gramática específica para realizar a simulação (adaptação do L-Systems), deve-se conhecer profundamente o modelo a ser simulado. Para melhor entendimento do modelo, tem-se a fenologia (estudo do ciclo das culturas ao longo do processo de desenvolvimento e contribui para o entendimento de suas diferentes fases: germinação, emergência, crescimento e desenvolvimento vegetativo, florescimento, frutificação, formação das sementes e maturação). Isso torna possível identificar as relações e as influências dos fatores envolvidos no processo de produção, favorecendo a previsão de problemas e a tomada de decisão. No entanto, apenas uma simulação do crescimento/desenvolvimento de uma cultura não é suficiente para atender aos anseios de um discente/docente que estuda uma determinada variedade vegetal. É necessário que o simulador (AV) também tenha uma capacidade reativa (interação) que demonstre os resultados da interferência de variáveis (macronutrientes) na escala fenológica de uma planta. Ou seja, as características do solo podem ser verificadas na relação com uma cultura, especificamente neste caso a soja, propiciando um 
VI Congresso Brasileiro de Informática na Educação (CBIE 2017)

Anais do XXVIII Simpósio Brasileiro de Informática na Educação (SBIE 2017)

resultado visual/gráfico por meio de um ambiente virtual de simulação. Para realizar a avaliação do ManjeoSoja3D (ambiente virtual criado para atingir estes objetivos e resolver os problemas apresentados).

Os primeiros modelos que foram capazes de realizar a simulação de crescimento e produção de culturas surgiram na década de 70 (JONES; KEATING; PORTER, 2001). A partir destes modelos foi possível integrar os conhecimentos dos processos biofísicos que regem no sistema solo-água-planta, permitindo identificar e avaliar as incertezas na produção associadas às diferentes opções de manejo (ação de conjugar as atividades manuais com a tecnologia avançada, com o objetivo de definir ou traçar decisões a serem tomadas em um sistema de produção agropecuário (CAMILO et al., 2013), (EMBRAPA, Marin, 2006).

Para realizar a simulação de crescimento da soja de forma autônoma é preciso empregar algum formalismo matemático. Em 1968, o biólogo Aristid Lindenmayer introduziu um formalismo do desenvolvimento celular de pequenos organismos multicelulares (LINDENMAYER, 1968), baseada nas gramáticas de Chomsky e sistemas de reescrita, posteriormente denominado LSystems (PRUSINKIEWICZ et al., 1996). Logo após, PRUSINKIEWICZ propôs a interpretação gráfica das gramáticas L-systems, tal abordagem assume que determinados símbolos existentes em uma cadeia produzida por uma gramática L-system podem reproduzir uma forma gráfica, assim possibilitando reproduzir em forma gráfica o crescimento de uma planta.

Além de uma gramática específica (formalismo matemático), para realizar a simulação deve-se conhecer profundamente o modelo a ser simulado. Para melhor entendimento do modelo de crescimento da cultura da soja, tem-se a fenologia, que estuda o ciclo das culturas ao longo do processo de desenvolvimento e contribui para o entendimento de suas diferentes fases: germinação, emergência, crescimento e desenvolvimento vegetativo, florescimento, frutificação, formação das sementes e maturação. Isso torna possível identificar as relações e as influências dos fatores envolvidos no processo de produção, favorecendo a previsão de problemas e a tomada de decisão. A escala fenológica, portanto, constitui-se em um conjunto de fases que descrevem o crescimento e o desenvolvimento de uma cultura desde sua emergência até a maturação, de forma a facilitar a sua compreensão (CAMILO, 2013).

No entanto, apenas uma simulação do crescimento/desenvolvimento de uma cultura não é suficiente para atender aos anseios de um pesquisador ou estudioso de uma determinada variedade vegetal. É necessário que o simulador (sistema) também tenha uma capacidade reativa (interação) que proporcione demonstrar os resultados da interferência de variáveis (micro e macronutrientes) na escala fenológica de uma planta. Ou seja, as características do solo podem ser verificadas na relação com uma cultura, especificamente neste caso a soja, propiciando um resultado visual/gráfico por meio de um ambiente virtual de simulação.

Desta forma, o objetivo deste trabalho é implementar (ambiente virtual e o consequente formalismo matemático) o crescimento autônomo da cultura da soja, elencando as principais exigências nutricionais da soja, juntamente com os efeitos do déficit destes nutrientes construindo um mecanismo de interpretação de análise do solo de acordo com formulas existentes na literatura.

\section{Fenologia da cultura da soja}

A metodologia proposta por Fehr e Caviness (1977) divide os estádios de desenvolvimento da soja em: (a) estádios vegetativos e (b) estádios reprodutivos. Como podemos observar na Figura 1 , os estádios vegetativos são designados pela letra $\mathrm{V}$ e os reprodutivos pela a letra $\mathrm{R}$, e essas letras são seguidos por índices numéricos que identificam estádios específicos, com exceção dos estádios VE (emergência) e VC (cotilédone). Essa metodologia identifica precisamente o estádios de desenvolvimento em que se encontra uma planta ou lavoura de soja (FARIAS; NEPOMUCENO; NEUMAIER, 2007). 
VI Congresso Brasileiro de Informática na Educação (CBIE 2017)

Anais do XXVIII Simpósio Brasileiro de Informática na Educação (SBIE 2017)

\begin{tabular}{|c|c|c|c|}
\hline \multicolumn{2}{|c|}{ Estádios Vegetativos } & \multicolumn{2}{|r|}{ Estádios Reprodutivos } \\
\hline VE & Emergência & R1 & Inicio do Florescimento \\
\hline vc & Cotilêdone & R2 & Florescimento Pleno \\
\hline V2 & Segundo Nó & R3 & Inicio da Formação do Legume \\
\hline v4 & Quarto Nó & R4 & Legume Completamente Desenvolvido \\
\hline \multirow[t]{4}{*}{ VN } & Enésimo Nó & R5 & Enchimento de Grão \\
\hline & & R6 & Grão Cheio ou Completo \\
\hline & & R7 & Início da Maturação \\
\hline & & $\mathbf{R 8}$ & Maturação Plena \\
\hline
\end{tabular}

Grãos dentro da vagem
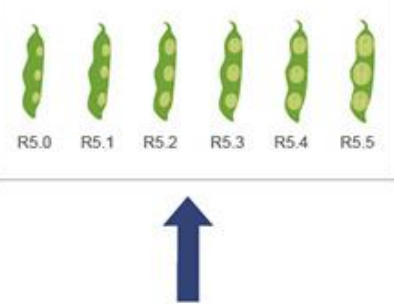

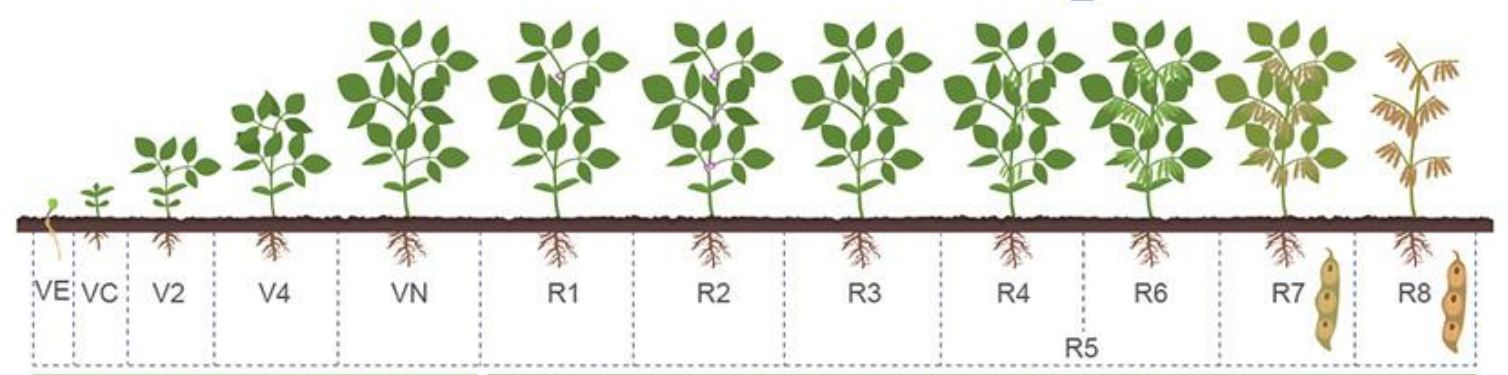

Estádios vegetativos

Estádios reprodutivos

Figura 1 - Estádios fenológicos da soja (Glycine max (L.) Merrill).

\subsection{Exigências Nutricionais da Soja}

A soja requer 16 elementos químicos para o seu desenvolvimento, dentre eles o carbono (C), hidrogênio $(\mathrm{H})$ e o oxigênio $(\mathrm{O})$, que são nutrientes encontrados na atmosfera e na água e participam da fotossíntese (SOUZA, 2014 apud SILVA, 2005). Além do C, H e O, treze outros elementos são fornecidos pelo solo, onde seis são requeridos em quantidade maiores chamados macronutrientes e outros setes requeridos em quantidade menores, chamados micronutrientes.

Este trabalho tem como objetivo avaliar o impacto dos macronutrientes, sendo assim, não haverá abordagem de todos os micronutrientes, apenas o Alumínio( $\mathrm{Al})$, não diminuindo a importância dos micronutrientes para o desenvolvimento da soja. O cálcio $(\mathrm{Ca})$, magnésio $(\mathrm{Mg})$, fósforo $(\mathrm{P})$, potássio $(\mathrm{K})$ e o enxofre $(\mathrm{S})$ fazem parte do grupo dos macronutrientes, dessa forma, suas principais funções e sintomas de deficiência são:

- Cálcio (Ca) - o cálcio é benéfico na germinação, ativa enzimas relacionadas ao metabolismo do fósforo e atua na manutenção da parede celular, dando um aspecto de turgidez e resistência à planta.

- Magnésio (Mg) - o magnésio é o átomo central da molécula de clorofila, fazendo parte na sua composição química (estrutura) e sendo fundamental nos processos da fotossíntese. No caso de sua deficiência, as folhas mais velhas apresentam clorose inter nerval, meio amarelada, e nervuras com cor verde-pálida.

- Potássio (K) - o potássio é o segundo elemento mais absorvido pela planta, pois é o principal ativador enzimático e regulador dos estômatos, favorecendo a retenção das vagens durante sua formação, reduz a deiscência na maturação e aumenta a resistência das plantas às doenças.

- Fósforo (P) - o fósforo é crucial no metabolismo das plantas, desempenhando papel importante na transferência de energia da célula, na respiração e na fotossíntese e absorção dos nutrientes do solo (ARANTES; SOUZA, 1993).

- Enxofre (S) - o enxofre pode ser absorvido pelas plantas através do oxigênio pelas folhas. Contudo, a fonte mais importante de enxofre é o sulfato absorvido pelas raízes da soja. O S contribui na composição dos aminoácidos e proteínas, sendo um nutriente importante para a transferência de eletro na fotossíntese, na sua falta, ocorre uma serie de 
VI Congresso Brasileiro de Informática na Educação (CBIE 2017)

Anais do XXVIII Simpósio Brasileiro de Informática na Educação (SBIE 2017)

distúrbios metabólicos, inclusive a diminuição da fotossíntese e respiração (ARANTES; SOUZA, 1993).

\section{L-Systems}

Em 1968, o biólogo Aristid Lindenmayer introduziu um formalismo do desenvolvimento celular de pequenos organismos multicelulares (LINDENMAYER, 1968), baseada nas gramáticas de Chomsky e sistemas de reescrita, posteriormente denominado L-Systems (PRUSINKIEWICZ et al., 1996). Segundo Santos (2008), o formalismo de Lindenmayer permite, por meio de uma gramática especial, a representação da reescrita paralela e simultânea, o que não ocorre nas gramáticas convencionais de Chomsky em que a reescrita ocorre sequencialmente. O processo de reescrita paralela e simultânea é a característica que permite aos L-systems representar objetos que apresentem auto-similaridade, como fractais e plantas.

O L-Systems é definido como uma gramática representada por uma tupla ordenada, $\mathrm{G}$ $=\mathrm{V}, \mathrm{T}, \mathrm{P}$, em que $\mathrm{V}$ representa um alfabeto de símbolos que podem ser usados para fazer sequencia de caracteres, T como uma string inicial denominado "axioma" por onde começa a construção da sequencia e P como uma coleção de regras de produção que expandem cada símbolo em uma sequência maior de símbolos. A Figura 2 demonstra um exemplo desta gramática.

A ideia básica desta interpretação geométrica por meio da gramática do L-Systems é dada por um cursor gráfico capaz de produzir traçados geométricos em coordenadas cartesianas mediante a alteração de estado da máquina que o controla. O estado desse cursor é alterado mediante a troca de valores da tripla $(\boldsymbol{x}, \boldsymbol{y}, \boldsymbol{a})$, sendo o par $(\boldsymbol{x}, \boldsymbol{y})$ a coordenada atual desse cursor a partir do qual será calculada, pelas relações trigonométricas do triângulo retângulo, a nova posição $\left(\boldsymbol{x}^{\prime}, \boldsymbol{y}^{\prime}\right)$ dado o valor de deslocamento $\boldsymbol{d}$ (passos) desejado para o cursor. Além disso, $\boldsymbol{a}$ é o ângulo que determinará a orientação incremental no qual se dará o deslocamento.

\begin{tabular}{|c|c|c|}
\hline Definição & Interação & Produção \\
\hline \multirow{5}{*}{$\begin{array}{l}\text { a) } V=\{S, A, B\} \\
T=\{\} \\
P=\{S \rightarrow A, \\
A \rightarrow A B, \\
B \rightarrow A\}\end{array}$} & $\mathbf{n}=\mathbf{0}$ & A \\
\hline & $\mathbf{n}=\mathbf{1}$ & AB \\
\hline & $\mathbf{n}=\mathbf{2}$ & ABA \\
\hline & $\mathbf{n}=3$ & ABAAB \\
\hline & $n=4$ & ABAABABA \\
\hline \multirow{4}{*}{$\begin{array}{l}b) a=25 \\
V=\{S, F\} \\
T=\{S\} \\
P=\{S \rightarrow F \\
F \rightarrow F /+F\rangle / F / F\}\end{array}$} & $\mathbf{n}=\mathbf{0}$ & $\mathbf{F}$ \\
\hline & $\mathbf{n}=\mathbf{1}$ & $\mathbf{F}[+\mathbf{F}] \mathbf{F}[-\mathbf{F}] \mathbf{F}$ \\
\hline & $\mathbf{n}=\mathbf{2}$ & $\begin{array}{l}\mathbf{F}[+\mathbf{F}] \mathbf{F}[-\mathbf{F}] \mathbf{F}[+\mathbf{F}[+\mathbf{F}] \mathbf{F}[-\mathbf{F}] \mathbf{F}] \mathbf{F}[+\mathbf{F}] \mathbf{F}[-\mathbf{F}] \\
\mathbf{F}[-\mathbf{F}[+\mathbf{F}] \mathbf{F}[-\mathbf{F}] \mathbf{F}] \mathbf{F}[+\mathbf{F}] \mathbf{F}[-\mathbf{F}] \mathbf{F}\end{array}$ \\
\hline & $\mathbf{n}=\mathbf{3}$ & $\begin{array}{l}\mathbf{F}[+\mathbf{F}] \mathbf{F}[-\mathbf{F}] \mathbf{F}[+\mathbf{F}[+\mathbf{F}] \mathbf{F}[-\mathbf{F}] \mathbf{F}] \mathbf{F}[+\mathbf{F}] \mathbf{F}[-\mathbf{F}] \mathbf{F}[- \\
\mathbf{F}[+\mathbf{F}] \mathbf{F}[-\mathbf{F}] \mathbf{F}] \mathbf{F}[+\mathbf{F}] \mathbf{F}[-\mathbf{F}] \mathbf{F}[+\mathbf{F}[+\mathbf{F}] \mathbf{F}[- \\
\mathbf{F}] \mathbf{F}[+\mathbf{F}[+\mathbf{F}] \mathbf{F}[-\mathbf{F}] \mathbf{F}] \mathbf{F}[+\mathbf{F}] \mathbf{F}[-\mathbf{F}] \mathbf{F}[-\mathbf{F}[+\mathbf{F}] \mathbf{F}[- \\
\mathbf{F}] \mathbf{F}] \mathbf{F}[+\mathbf{F}] \mathbf{F}[-\mathbf{F}] \mathbf{F}] \mathbf{F}[+\mathbf{F}] \mathbf{F}[-\mathbf{F}] \mathbf{F}[+\mathbf{F}[+\mathbf{F}] \mathbf{F}[- \\
\mathbf{F}] \mathbf{F}] \mathbf{F}[+\mathbf{F}] \mathbf{F}[-\mathbf{F}] \mathbf{F}[-\mathbf{F}[+\mathbf{F}] \mathbf{F}[-\mathbf{F}] \mathbf{F}] \mathbf{F}[+\mathbf{F}] \mathbf{F}[- \\
\mathbf{F}] \mathbf{F}[-\mathbf{F}[+\mathbf{F}] \mathbf{F}[-\mathbf{F}] \mathbf{F}[+\mathbf{F}[+\mathbf{F}] \mathbf{F}[-\mathbf{F}] \mathbf{F}] \mathbf{F}[+\mathbf{F}] \mathbf{F}[- \\
\mathbf{F}] \mathbf{F}[-\mathbf{F}[+\mathbf{F}] \mathbf{F}[-\mathbf{F}] \mathbf{F}] \mathbf{F}[+\mathbf{F}] \mathbf{F}[-\mathbf{F}] \mathbf{F}] \mathbf{F}[+\mathbf{F}] \mathbf{F}[- \\
\mathbf{F}] \mathbf{F}[+\mathbf{F}[+\mathbf{F}] \mathbf{F}[-\mathbf{F}] \mathbf{F}] \mathbf{F}[+\mathbf{F}] \mathbf{F}[-\mathbf{F}] \mathbf{F}[-\mathbf{F}[+\mathbf{F}] \mathbf{F}[- \\
\mathbf{F}] \mathbf{F}] \mathbf{F}[+\mathbf{F}] \mathbf{F}[-\mathbf{F}] \mathbf{F}\end{array}$ \\
\hline
\end{tabular}

Figura 2 - Exemplo de gramática L-Systems.

Desta forma, a principal contribuição deste trabalho é a construção de um ambiente virtual que simule o desenvolvimento fenológico da soja adaptando a gramática L-Systems para simular o crescimento e a interferência dos macronutrientes.

\section{Trabalhos Relacionados}


VI Congresso Brasileiro de Informática na Educação (CBIE 2017)

Anais do XXVIII Simpósio Brasileiro de Informática na Educação (SBIE 2017)

O método utilizado para a busca dos trabalhos apresentados consistiu em aplicar a uma string (animation, simulation, visual models, plant growth, tree growth, virtual reality) em bases referenciais importantes oferecidos pelas bases IEEE Xplorer1, ACM Digital Library2, Scopus3, Google Scholar e portal EMBRAPA. A fim de obter publicações que não foram recuperadas pelas máquinas de busca e que sejam importantes para este trabalho, foi feita uma análise manual das referências dos artigos retornados por essas fontes encontradas previamente.

A Tabela 1 apresenta um conjunto de trabalhos que foram analisados para dar sustentação científica (análise de relevância e contribuição) a construção desta proposta. Os trabalhos foram: T1 (Capturing and Animating the Morphogenesis of Polygonal Tree Models (PIRK et al., 2012)), T2 (LiveTree: Realistic tree growth simulation tool (KIM; JEONG, 2012)), T3 (Study on Method of Modeling and Visualization of Soybean (ZHONGBIN et al., 2006)), T4 (Digital Design and implementation of Soybean Growth process based on L-System (ZHONGBIN et al., 2006)) e T5 (DSSAT cropping system model (JONES et al., 2013)).

\begin{tabular}{lccccc}
\hline & C1(L-Systems) & C2(Modelo) & C3(Metodologia) & C4(Visualização) & C5(SAD) \\
\hline T1 & Sim & Autônomo & Árvores de grande porte & 3D & Não \\
\hline T2 & Não & Estático & Árvores de grande porte & 3D & Não \\
\hline T3 & Sim & Autônomo & Específica da soja & 3D & Não \\
\hline T4 & Sim & Autônomo & Específica da soja & 3D & Não \\
\hline T5 & Não & Autônomo & Qualquer espécie & Gráfico (2D) & Sim \\
\hline
\end{tabular}

Tabela 1 - Comparativos entre trabalhos analisados.

\section{Arquitetura e Implementação}

Para propiciar a criação de um sistema que se baseasse em uma gramática (formalismo) e com uma adição de funcionalidades específicas que interferisse no modelo de crescimento foram criados os seguintes módulos: a) crescimento natural (regra baseada na gramática do L-System); b) fenologia (regras para que o crescimento tenha obediência as respectivas fases de crescimento da soja); c) renderização 3D (adaptação ao L-System e às variáveis da composição do solo); d) população (análise de indivíduo ou de população).

O ambiente virtual foi desenvolvido com uso do motor de jogo, Unity, o qual permite a modelagem do terreno, criação de objetos 3D, interação com o usuário entre outras coisas e a linguagem de programação utilizada na Unity, para implementação da interação com o usuário é o C\# (C Sharp). No ambiente virtual o usuário pode optar entre duas formas de visualização da soja: a) individual (Figura 3): somente uma planta é apresentada para realizar a simulação e b) população: onde varias plantas são agrupadas em população para simular uma plantação real de soja.

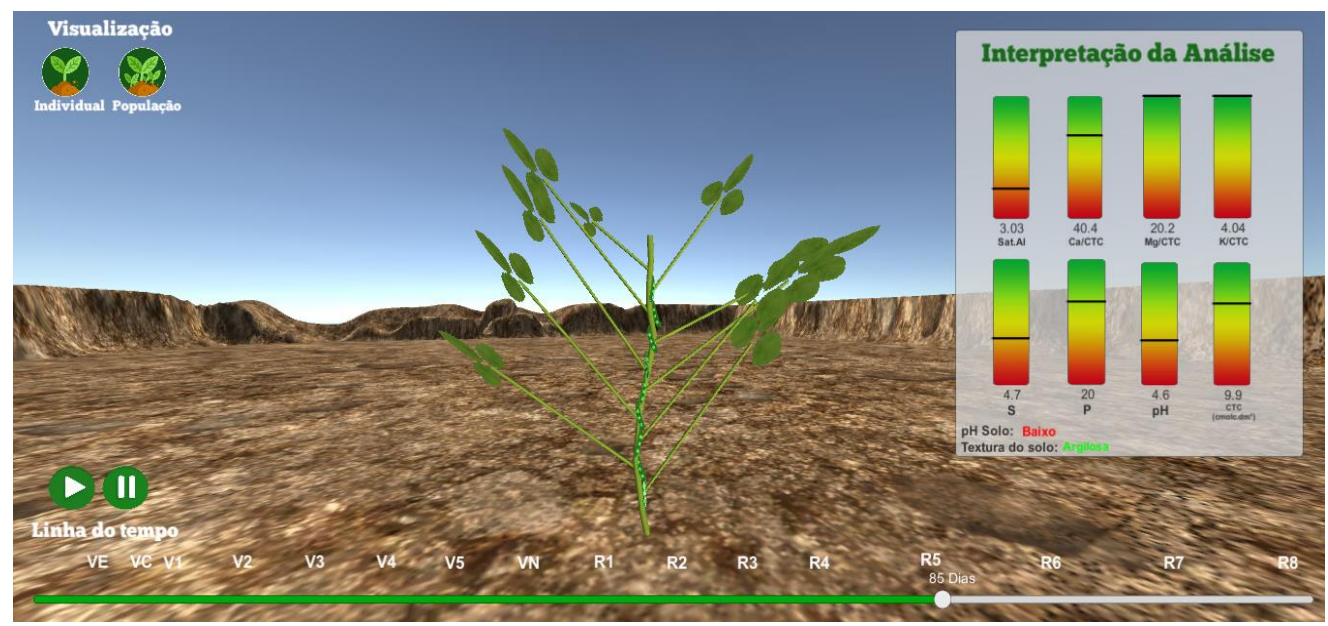

Figura 3 - Interface gráfica do sistema (visão de um indivíduo). 
VI Congresso Brasileiro de Informática na Educação (CBIE 2017)

Anais do XXVIII Simpósio Brasileiro de Informática na Educação (SBIE 2017)

O principal algoritmo do sistema (em forma de pseudo-código) apresenta regras e a consequente implementação destas regras transformando os dados em imagem, este algoritmo está representado abaixo:

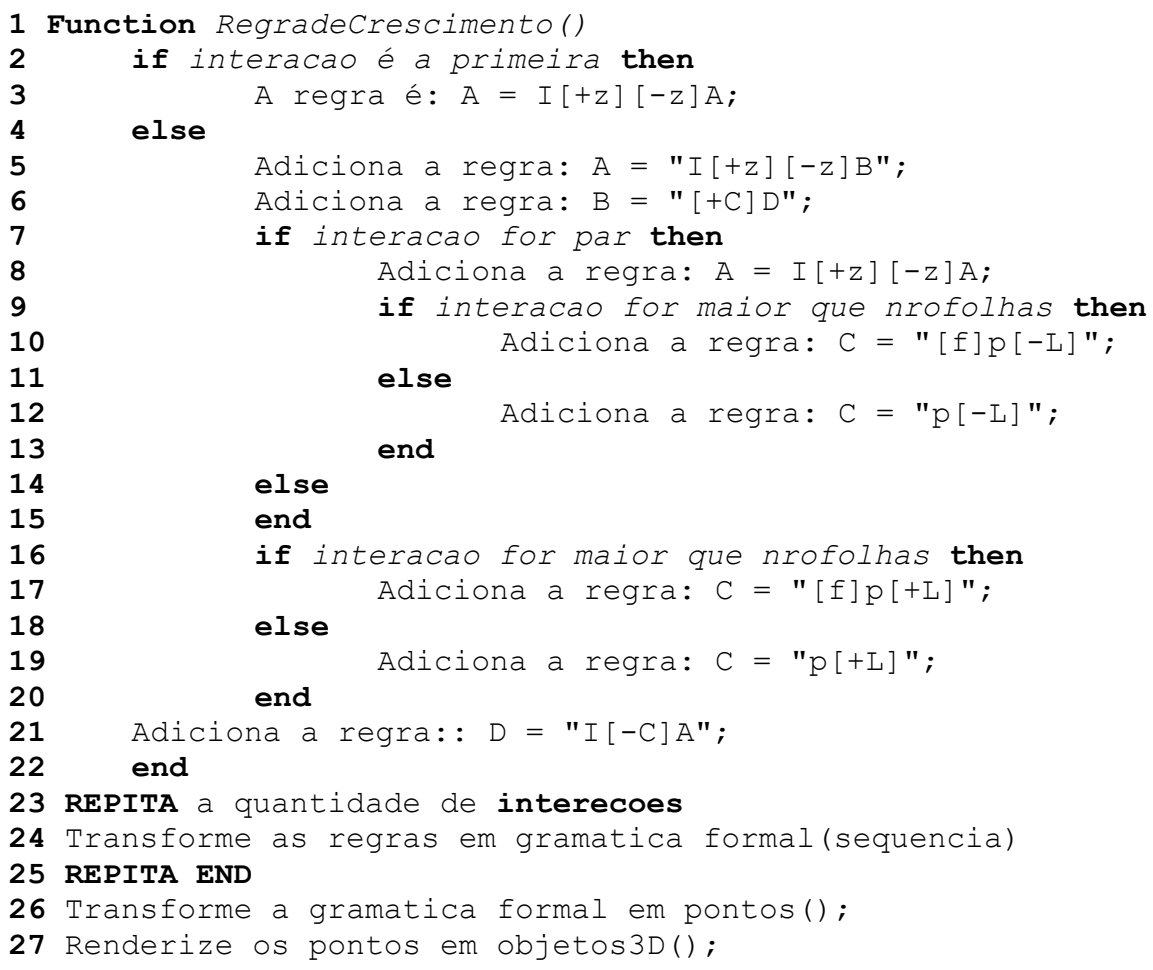

\section{Funcionamento do Sistema}

Dentre as duas formas de visualização providas pelo Soja3D, na forma individual, somente é possível realizar a simulação com uma planta, sendo que a câmera é fixa e focada na planta. Em contrapartida, na forma de visualização em população é possível navegação pelo ambiente, acompanhando várias plantas pelo terreno, possibilitando observar mais de perto o crescimento da lavoura como um todo. A Figura 4 apresenta a interface do sistema e os respectivos nutrientes de uma análise.

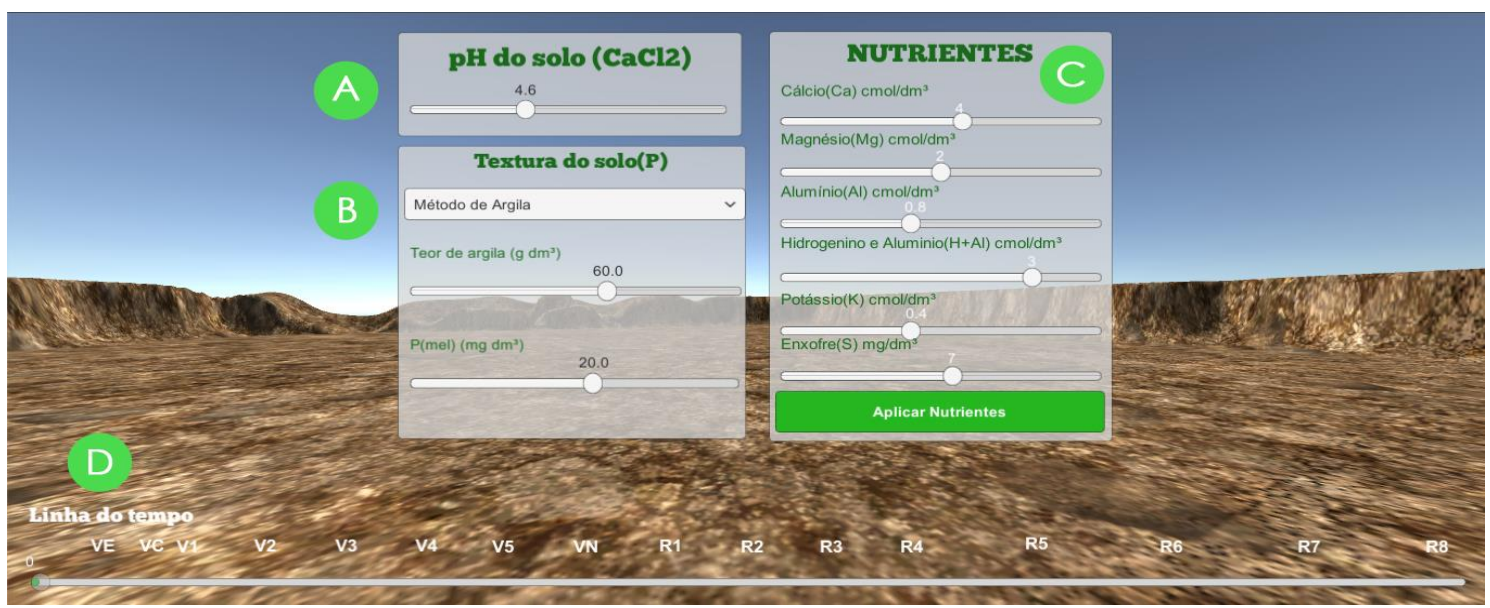

Figura 4 - Insersão dos nutrientes de uma análise de solo.

Já a Figura 5 apresenta a interface do sistema com uma população de plantas. Enquanto que a Figura 6 ilustra plantas com deficiências nutricionais. 
VI Congresso Brasileiro de Informática na Educação (CBIE 2017)

Anais do XXVIII Simpósio Brasileiro de Informática na Educação (SBIE 2017)

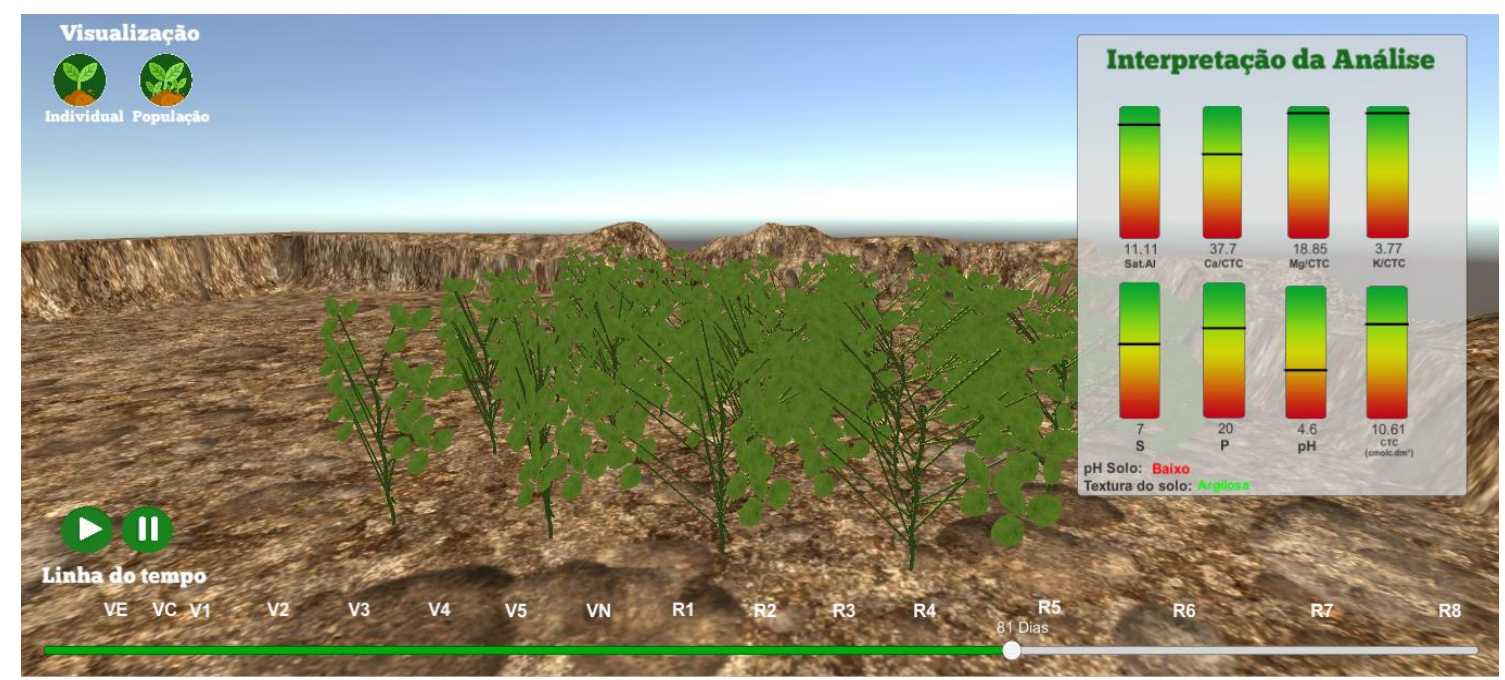

Figura 5 - População de plantas.

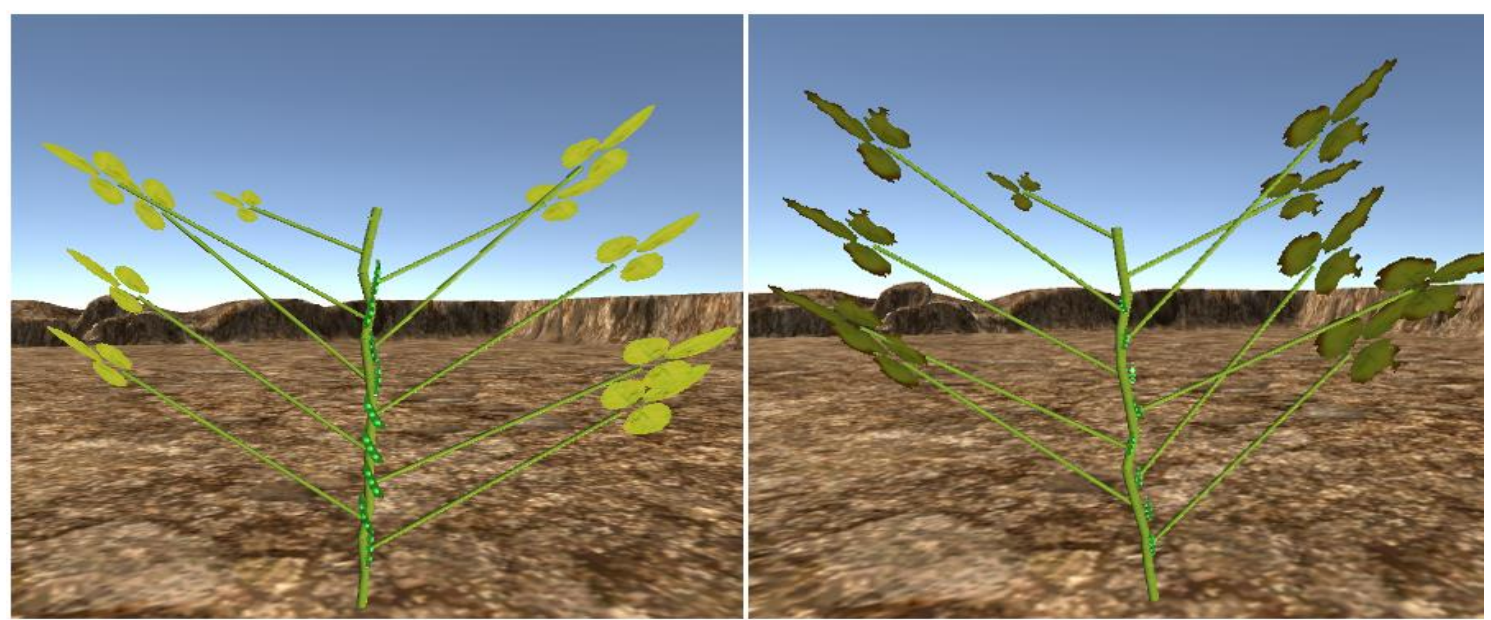

Figura 6 - Plantas com deficiência de nutrientes.

\section{Análise e Testes}

Para realizar a avaliação do Soja3D, foi utilizado um dos mais conhecidos e mais simples métodos de averiguação do nível de usabilidade de um sistema, denominado: System Usability Scale (SUS), proposto por Brooke et al., 1996. O SUS pode ser utilizado para avaliar aplicação, software, websites, serviços, hardware e qualquer outro tipo de interface. O SUS permite avaliar os seguintes critérios:

- Efetividade (os usuários conseguem completar seus objetivos?)

- Eficiência (quanto esforço e recursos são necessários para isso?)

- Satisfação (a experiência foi satisfatória?)

O SUS se resume em 10 perguntas, e para cada uma delas o usuário pode responder em uma escala de variando de 1 a 5, onde 1 significa "Discordo Completamente" e 5, significando "Concordo Completamente". Essa mesma escala também foi utilizada para responder outras questões da categoria "conhecimento" e "corretude" que serão apresentadas logo abaixo.

Em conjunto com o modelo proposto por Brooke et al., 1996, foi utilizado o paradigma Goal/Question/Metric (GQM) (CALDIERA; ROMBACH, 1994), que consiste em uma abordagem orientada a objetivos para medição de produtos e processos de software. De acordo com Caldiera e Rombach (1994) o GQM é dividido em três partes, sendo elas: a) 
VI Congresso Brasileiro de Informática na Educação (CBIE 2017)

Anais do XXVIII Simpósio Brasileiro de Informática na Educação (SBIE 2017)

Nível conceitual (objetivos): nesse nível, deve-se definir quais metas pretende-se atingir; b) Nível operacional (questões): nesse nível é elaborado um conjunto de questões quantificáveis que especifiquem as medidas adequadas para sua avaliação; e c) Nível quantitativo (métricas): um conjunto de dados é associado a cada questão para que essa seja respondida de forma quantitativa.

Além das 10 perguntas básicas do método SUS, foram realizadas outras 6 questões referentes as categorias "conhecimento adquirido" e "corretude" dos conceitos aplicados no sistema.

Baseado no modelo para avaliação da usabilidade proposto por Brooke et al., 1996 e na abordagem GQM, o modelo de avaliação do sistema proposto neste trabalho consiste dos seguintes objetivos, questões e métricas:

1. Objetivo 1 : Analisar o Soja3D com o propósito de verificar a usabilidade.

a. Questão: Qual a pontuação obtida pelo método SUS?

b. Métricas

i. \#MEDIAOK: a pontuação média obtida no SUS é maior ou igual a 68 pontos.

ii. \#MEDIAFRACA: a pontuação média obtida no SUS é menor a 68 pontos.

2. Objetivo 2 : Analisar o Soja3D com o proposito de verificar a utilidade no processo de ensino/aprendizado dos conceitos de análise e interpretação de solo.

a. Questão: O Soja3D é útil no processo de ensino/aprendizado dos conceitos de análise e interpretação de solo?

b. Métricas:

i. \#CONHECIMENTOOK: porcentagem de respostas do tipo "Concordo parcialmente" ou "Concordo totalmente" para todas as questões da categoria "conhecimento adquirido".

ii. \#CONHECIMENTOFRACO: porcentagem media de respostas do tipo "Discordo parcialmente" ou "Discordo totalmente" para todas as questões da categoria "conhecimento adquirido".

3. Objetivo 3 : Analisar o Soja3D com o proposito de verificar se conceitos de interpretação da análise de solo e fenologia no Soja3D foram abordados de forma correta?

a. Questão: Os conceitos de interpretação da analise de solo e da fenologia da soja no Soja3D condizem com a realidade?

b. Métricas:

i. \#CORRETUDEOK: porcentagem media de respostas do tipo "Concordo parcialmente" ou "Concordo totalmente" para todas as questões da categoria "corretude".

ii. \#CORRETUDEFRACA: porcentagem media de respostas do tipo "Discordo parcialmente" ou "Discordo totalmente" para todas as questões da categoria "corretude".

Interpretação das métricas: Quanto ao Objetivo1 (usabilidade), se a métrica obtida for \#MEDIAOK o sistema proposto possui a facilidade de ser claramente compreendido e 
VI Congresso Brasileiro de Informática na Educação (CBIE 2017)

Anais do XXVIII Simpósio Brasileiro de Informática na Educação (SBIE 2017)

manipulado pelo usuário.

Para coletar as métricas deste modelo de avaliação, foram considerados três grupos diferentes de avaliadores (G1\{Engenheiro Agrônomos\}, G2\{Acadêmicos do Curso de Agronomia\} e G3\{Acadêmicos do Curso de Ciências da Computação\}), segundo Nielsen (1994), grupos de avaliadores devem ser formados por, no mínimo, três indivíduos e, no máximo, por cinco indivíduos.

A primeira métrica proposta pelo modelo GQM é avaliar a usabilidade do sistema. Como podemos observar na Figura 7 a média obtida pelo método SUS é 76,66 pontos, com isso concluímos que a métrica \#MEDIAOK foi atingida, comprovando a facilidade de uso pelos engenheiros agrônomos.

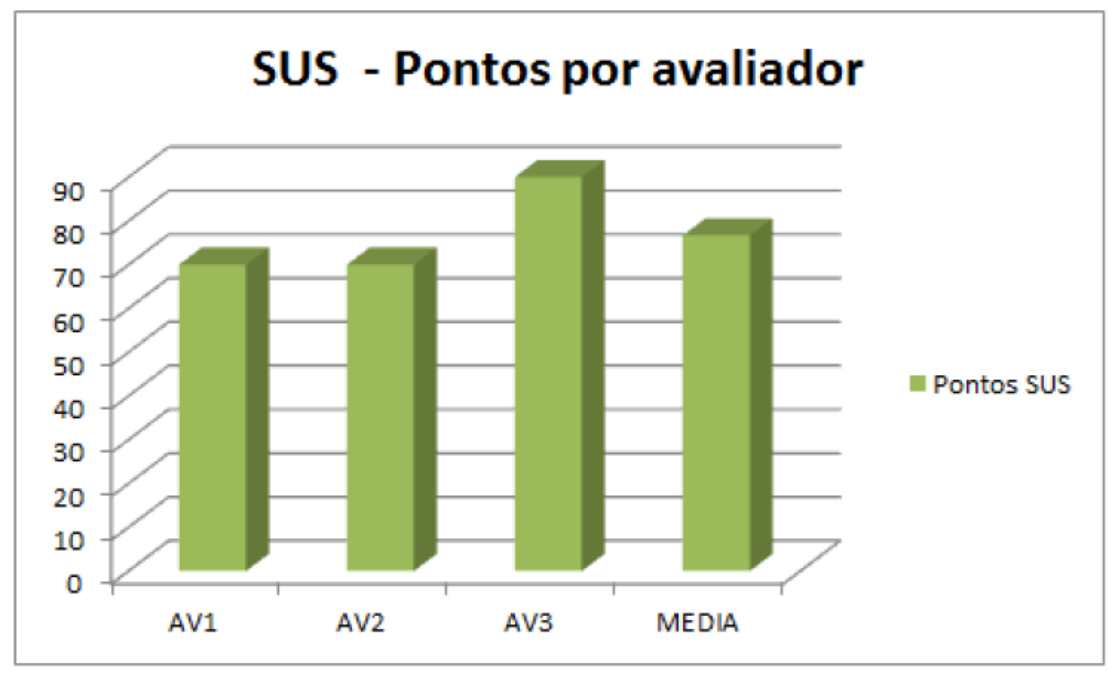

Figura 7 Média de pontos obtida pelo método SUS, avaliação feita pelos engenheiros agrônomos.

A Segunda métrica proposta pelo GQM é avaliar se o sistema foi útil no processo de ensino/aprendizado dos conceitos de análise e interpretação de solo. Com base nos valores apresentados na Figura 8 é possível observar as questões pertinentes a categoria conhecimento adquirido (Q11, Q12, Q13) e concluir que a métrica \#CONHECIMENTOOK também foi atingida com a porcentagem de $77,33 \%$ de respostas "Concordo Totalmente", comprovando a aquisição de conhecimento por parte dos engenheiros agrônomos.

A Terceira métrica tem o intuito de avaliar se os conceitos de interpretação da análise de solo e fenologia no Soja3D foram abordados de forma correta. Se as questões Q14, Q15 e Q16 da Figura 9 forem analisadas é possível chegar à conclusão que a métrica \#CORRETUDEOK também foi atingida com a porcentagem de $67 \%$, confirmando que o conceitos foram empregados de forma correta.
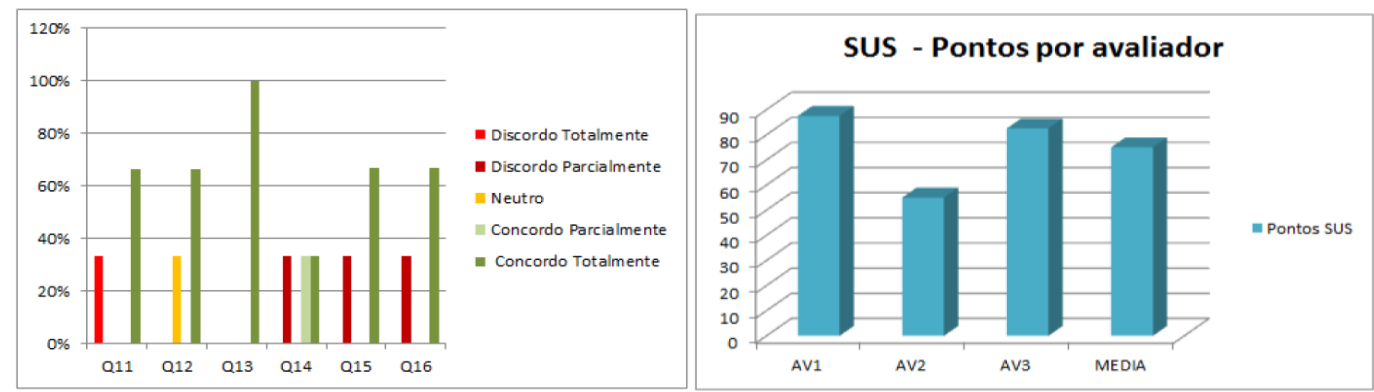

Figuras 8/9 Avaliação com agrônomos. / Avaliação pelo método SUS. 
VI Congresso Brasileiro de Informática na Educação (CBIE 2017)

Anais do XXVIII Simpósio Brasileiro de Informática na Educação (SBIE 2017)

Como base na Figura 9 a qual apresenta o resultado da avaliação utilizando o método SUS, é possível chegar à conclusão que a métrica \#MEDIAOK foi atingida com a média de 75 pontos, afirmando que o sistema apresenta usabilidade.

\section{Conclusões e Trabalhos Futuros}

O agronegócio vem mostrando crescimentos extraordinários durante os últimos anos, a tecnologia em si está contribuindo diretamente para que isso aconteça, agregando praticidade e facilitando a execução de tarefas. Desta forma, a área de simulação computacional junto com a agricultura de precisão vem ganhando espaço no mercado, sendo considerado um sistema com o objetivo de reduzir os custos e aumentar a produtividade. A maioria das ferramentas existentes no mercado para essa finalidade não são intuitivas, pois utilizam gráficos e tabelas para apresentar visualização da informação e muita das vezes estão em língua estrangeira dificultando ainda mais o entendimento. Existe também uma dificuldade de ensinar e aprender os conceitos de crescimento da soja e interpretação da análise de solo, pois, o que é empregado nos livros já está defasado e muitas das vezes não condizem com a realidade, para isso é preciso realizar o plantio e aulas de campos para elucidar esses conceitos. Esse problema pode ser parcialmente solucionado pôr o ambiente virtual resultante desse trabalho, pois ele proporciona uma alternativa ao modelo tradicional de aquisição do conhecimento e a interpretação das análise de solo.

A criação de uma aplicação que integra crescimento autônomo de plantas em 3D, interpretação de análise de solo e realidade virtual para a área agronômica reforça ainda mais importância de ferramentas computacionais no ensino/aprendizagem e apoio a decisão. $\mathrm{O}$ Soja3D abordou somente a simulação de crescimento da soja, podendo ser adaptada para qualquer outra cultura, como milho, algodão, eucalipto. Assim sugerindo o desenvolvimento de novas pesquisas nesse sentido.

Como parte dos trabalhos futuros, pretende-se tornar mais realístico o crescimento tridimensional da soja, para isso, é preciso melhorar a implementação do L-Systems e incluir fatores externos como sol, chuva, vento e micronutrientes. Outro trabalho futuro é implementar o cálculo da recomendação de adubação para os nutrientes que apresentarem valores baixos e também realizar estimativa de produção de grãos.

\section{Referências}

Arantes, Neylson Eustáquio, and Plínio Itamar de Mello de Souza. Cultura da soja nos cerrados. Associação Brasileira para Pesquisa da Potassa e do Fofato, 1993.

Brooke, John. "SUS-A quick and dirty usability scale." Usability evaluation in industry 189.194 (1996): 4-7.

Caldiera, Victor R. Basili-Gianluigi, and H. Dieter Rombach. "Goal question metric paradigm." Encyclopedia of Software Engineering 1 (1994): 528-532.

Camilo, Yanuzi Mara Vargas, et al. "Fenologia, produção e precocidade de plantas de Eugenia dysenterica visando melhoramento genético." Revista de Ciências Agrárias 36.2 (2013): 192-198.

EMBRAPA, Marin. Fenologia EMBRAPA. 2006. <http://www.agencia.cnptia.embrapa. br/gestor/cana-de-acucar/arvore/CONTAG01_68_22122006154840.html >. Online; Acessado em: 24/07/2016.

FARIAS, José Renato B., Alexandre L. NEPOMUCENO, and Norman NEUMAIER. "Ecofisiologia da soja." Embrapa Soja. Circular técnica (2007).

Fehr, W. R., and C. E. Caviness. "Stages of soybean development, Iowa State University, Special Report 80." Iowa State University, Ames (1977).

Jones, J. W., B. A. Keating, and C. H. Porter. "Approaches to modular model development." Agricultural Systems, Elsevier, v. 70.2 (2001): p. 421-443. 
VI Congresso Brasileiro de Informática na Educação (CBIE 2017)

Anais do XXVIII Simpósio Brasileiro de Informática na Educação (SBIE 2017)

Jones, James W., et al. "The DSSAT cropping system model." European journal of agronomy, Elsevier, v. 18.3 (2003): p. 235-265.

Kim, Jaehwan, and Il Kwon Jeong. "LiveTree: Realistic tree growth simulation tool." ACM SIGGRAPH 2012 Posters. ACM, 2012.

Lindenmayer, Aristid. "Mathematical models for cellular interactions in development I. Filaments with one-sided inputs." Journal of theoretical biology ,Elsevier, v. 18.3 (1968): p. 280-299.

NIELSEN, J. Usability inspection methods. In: ACM. Conference companion on Humanfactors in computing systems. [S.1.], 1994. p. 413414.

Pirk, Sören, et al. "Capturing and animating the morphogenesis of polygonal tree models." ACM Transactions on Graphics (TOG) v. 31.6 (2012): p. 169.

Prusinkiewicz, Przemyslaw. "Graphical applications of L-systems." Proceedings of graphics interface. Vol. 86. No. 86. 1986.

Prusinkiewicz, Przemyslaw, et al. "L-systems: from the theory to visual models of plants."

Proceedings of the 2nd CSIRO Symposium on Computational Challenges in Life Sciences. Vol. 3. 1996.

Silva, R. J. "Elementos essenciais: Química e fertilidade do solo.” Universidade Federal do Acre, 2005.

Souza, M. B. "Manejo adequado de macro e micronutrientes na cultura da soja em solos do cerrado" (2014).

ZHONGBIN, Su, et al. Study on Method of Modeling and Visualization of Soybean. In: Plant Growth Modeling and Applications, 2006. PMA'06. Second International Symposium on. IEEE, 2006. p. 289-294. 